Historic, Archive Document

Do not assume content reflects current scientific knowledge, policies, or practices. 



\subsection{1}

OUR SILENT SALESMAN
MCHUTCHISON \& CO.

Horticultural Merchants

95 CHAMBERS STREET

NEW YORK

IMMEDIATE SHIFMENT

The buyer and seller agrees that the seller gives no warranty, express or implied, as to the productiveness of the stock hereby offered, apd its retehtiou for len days after reckipt if the huser without complaint in writing. constitutes a definite aceptance thereof by the huser. All growing stock is offered subject to srop.
REPUTATION - yours and ours, depends on the QUALITY of the goods we (and you) sifl 11. Price is soon forgotten when satisfaction in quality is attained. In-tis orforing quality comes first - anybody can cut prices. We repeat the suggestion - "Don't starve your business - foed it and let it grow". Here's the menu:-

\section{FPETSIA BUIAS}

Improved Purity in good supply but going fast.

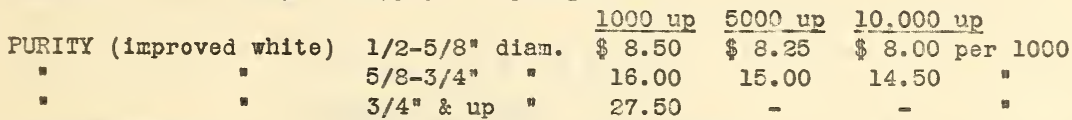

RAINBOW MIXTURE - all colors, 5/8-3/4" diam. \$3.00 per $100 \$ 25.00$ per 1000

\section{CAIIJA ROOTS}

This is one of our leaders - you get the benefit of carload freight rates to us in these prices:-

\begin{tabular}{|c|c|c|c|c|c|c|}
\hline & & & Per 100 & 1000 up & 5000 노 & \\
\hline GODFREY (smaller white) & $1-14^{n}$ & diameter & $\$ 8.00$ & $\$ 73.50$ & $\$ 70.00$ & per 1000 \\
\hline - & $1 \frac{1}{4}-1 \frac{1 / 2}{2}$ & 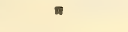 & 12.50 & 115.00 & 106.00 & " \\
\hline - & $1 \frac{1}{2}-13 / 4 \mathrm{~m}$ & - & 16.00 & 145.00 & - & - \\
\hline AETHIOPICA (large white) & $11 / 4-1 \frac{1}{2}$ & n & $\epsilon .25$ & 55.00 & 52.50 & - \\
\hline - & $1 \frac{1}{2}-13 / 4 \pi$ & " & 11.50 & 105.00 & 100.00 & - \\
\hline 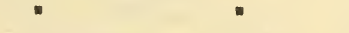 & $13 / 4=2$ & $n$ & 15.00 & 140.00 & - & - \\
\hline ELIIOTTIANA (yellow) & $1 \frac{1}{2}-2$ & $"$ & 11.00 & 100.00 & 95.00 & $n$ \\
\hline * n & $2-2 \frac{1}{2}$ & " & 14.50 & 135.00 & 130.00 & - \\
\hline - & $2 / 2-3$ & n & 20.00 & 185.00 & - & $"$ \\
\hline
\end{tabular}

Elliottiana - November shipment.

\section{IIIIIUM HARRISII}

Bermuda's finest type of high-grade bulbs

6-7 inch, packed 335 bulbs per case, $\$ 25.00$ per case

$7-9 " 200$ " $" 25.00$ "

\section{LILIUM CANDIDUM}

Madonna or St. Joseph's Lily. All Northern French grown, the hardy thickpetalled type. Blooms naturally in June - forces mell.

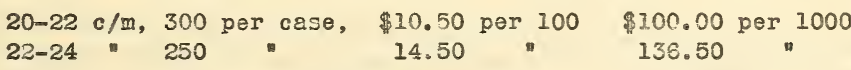

\section{CYCLAMEN SEEDS}

E. BIMPEWIES STRAIn only - in original packages.

\begin{tabular}{|c|c|c|c|c|}
\hline & 1000 & 5000 & 25,020 up & \\
\hline \#1 DARK BLOOD-RED $\ldots \ldots \ldots \ldots \ldots \ldots \ldots \ldots \ldots \ldots \ldots$ & $\$ 8.00$ & $\$ 7.75$ & $\$ 7.50$ per & 1000 \\
\hline 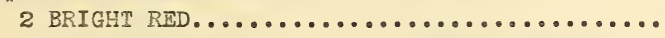 & 8.00 & 7.75 & 7.50 & n \\
\hline 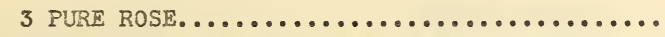 & 8.00 & 7.75 & 7.50 & $n$ \\
\hline 4 ROSE OF MAPIENTHAL, soft rose, carmine eye. & 8.00 & 7.75 & 7.50 & , \\
\hline 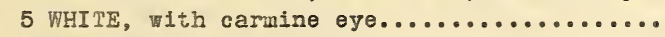 & 8.00 & 7.75 & 7.50 & n \\
\hline 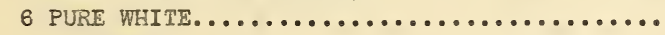 & 8.00 & 7.75 & 7.50 & " \\
\hline 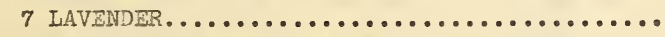 & 9.25 & 9.00 & 8.75 & $m$ \\
\hline 8 IMPROVED SAIMON, new, extra fine.......... & 11.00 & 10.50 & 10.25 & : \\
\hline 9 SATMON-SCARTET (Christmas Red), extra fine. & 12.50 & 12.00 & 11.75 & $=$ \\
\hline 10 PERTE OF ZELITNDORE; dark salmon, improved. & 10.00 & 9.75 & 9.50 & $n$ \\
\hline 11 ROSE OF ZEHLENDORF, light salmon.......... & 10.00 & 9.75 & 9.50 & . \\
\hline 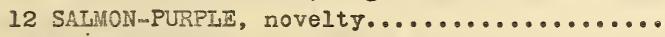 & 9.25 & 9.00 & 8.75 & 首 \\
\hline 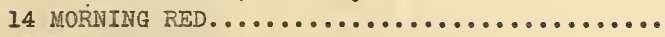 & 11.50 & 11.00 & 10.75 & - \\
\hline 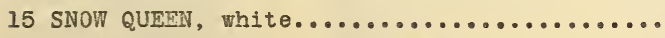 & 11.50 & 11.00 & 10.75 & - \\
\hline SAFFRON-RED, new, vary $f$ ino............. & 11.00 & 10.50 & 10.25 & - \\
\hline 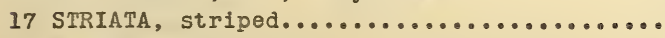 & 11.00 & 10.50 & 10.25 & $\bullet$ \\
\hline 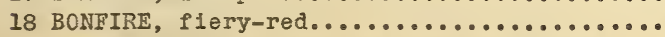 & 13.50 & 23.00 & 12.75 & 甲 \\
\hline
\end{tabular}




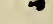




\title{
RUBBER PLANTS
}

FICUS ELASTICA, strong mossed rooted cuttings, $\$ 165.00$ per 1000

Packed in cases of 250 each, case lots only. F.O.B. Now

Orleans. Shipped promptly by express.

\section{ASPIDISTRAS} GREEN, established in $5 "$ pots, $\begin{gathered}10-14 \text { leaves each, } 81 / 4 \text { per leaf } \\ 2000 \text { leaves up } 8 \phi\end{gathered}$

F.O.B. New Orleans. Immediate shipment, by freight.

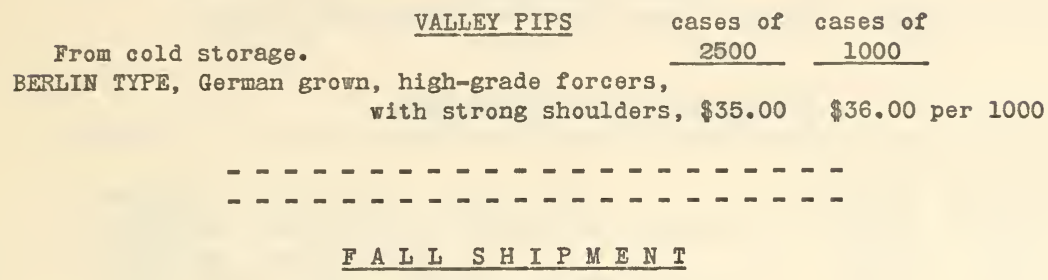

\section{JAPANESE IIIY BULBS (now crop)}

All bulbs thoroughly cured and ripened before leaving Japan. Uniformly graded and of the highest quality. For shipment on arrival we offer:-

\author{
IIIIUN FORMOSUM, all sizes, $\$ 17.50$ per case \\ " $\operatorname{ERABU}($ true) " 18.00 "
}

Discounts. Above prices are for 1 to 4 cases, 5 cases up $50 \phi$ less per case, 10 cases up $\$ 1.00$ less, discounts applying on single items, not totals.

\section{Case contents - all varieties}

\section{ERABU \& FORMOSUM}

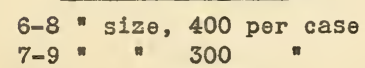

$8-10$ " 225 "

9-10" 200

$10-11$ " " 150

\section{GIGANTEUM}

$6-8$ "size, 400 por case

$7-9$ " " 300 "

8-10" " 225 "

9-10" 200 "

10-11" " 150 " and Springileld (III). Erabu and Formosum and Hardy varieties F.O.B. New York and Chicago. Harrisii, Sinensis, and Auratum Platyphyllum at New York only. Lower prices F.0.B. Seattle.

Prices upon Giganteum and Hardy varieties will be quoted firm about September. Moanwhile we are booking advance orders at open price subject to your acceptance of price when made, before delivery. Such advance orders always get a lower price than later buyers.

Storage. Erabu and Formosum should have at least a month's additional rest before you start them. Having cold storage facilities we can store the cases at $50 \%$ per case for first month and $20 \phi$ per case per month thereafter - but will ship out as soon as they arrive unless you instruct otherwise.

\section{IILIUM REGATE}

The Regal Lily (Hyriophyllum). Hardy, white, free bloomer. We offer only blooming sizes. Delivery September to spring, your option.

$\begin{array}{ccccc}\text { Large bulbs, } 4-5 \text { inches circ., } & \$ 11.00 & \text { per } 100 & \$ 105.00 \text { per } 1000 \\ 5-6 & \text { " } & 15.50 & 148.50\end{array}$

\section{PATM STIEDS}

We offer only imported fresh seeds, for delivery as they arrive.

$\begin{array}{lrr}\text { ARECA LUTESCENS } & \$ 40.00 \text { per } 10,000 & \$ 4.50 \text { per } 1000 \\ \text { COCOS WEDDELIANA } & 85.00 & 9.00 \\ \text { PHOENIX ROEBELENII } & 85.00 & 9.00 \\ \text { LATANIA BORBONICA } & 37.50 & 4.00 \\ \text { KENTIA FORSTERIANA (delivery Spring 1930) per bushel or per } 1000 \\ \quad \text { BELMOREANA }\end{array}$





\section{SANSEVIERIAS}

The demand still exceeds the supply and we have been refusing orders for months, but after completing booked orders we can ship limited quantities. Our plants are carefully selected and graded, shipped with mossed roots, by express.

$\begin{array}{llll}\text { LAURENTII (gold striped), } & 12-18 " & 2-5 & \text { leaves, } \$ 45.00 \\ 18-24 " & 3-6 & 60.00 \\ 24-36 " & 4-8 & \text { " } & 75.00\end{array}$

F.0.B. New York. F.0.B. Florida $\$ 5.00$ per 100 less.

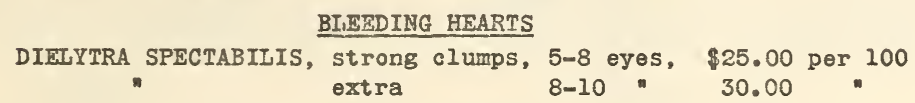

\section{IRIS BULBS (Tingitana Hybrids)}

Profitable to the grower who is equipped to force them. North-west grown.

WEDGEWOOD - the leading light blue

TINGITANA, THE FIRST - like Wodgewood but earlier

IMPERATOR - popular blue

YELIOW QUEEN - best tall dark yollow

GARNIER - long stemmed dark blue

D. HARING - uniform white, long stem

ADRIAN BACXER - lilac-purple, extra good

S. ROMBOUT - sky blue, long stem

D. VINCI - lemon yellow

DE WILDE - dark yello», shaded bronze

WHITE EXCELSIOR - best purest forcing white

GOLDEN GLORY - golden yellow

Orders for 5,000 up, less $5 \%$ special discount.

$\begin{array}{rrr}\frac{6-7 \mathrm{c} / \mathrm{m}}{\$ 112.00} & \frac{7-8 \mathrm{c} / \mathrm{m}}{\$ 140.00} \text { per } 1000 \\ 98.00 & 112.00 \\ 28.50 & 36.00 & \text { " } \\ 36.00 & 48.50 & " \\ 36.00 & 48.50 & \text { " } \\ 36.00 & 48.50 & \text { " } \\ 36.00 & 48.50 & \\ 36.00 & 48.50 & \text { " } \\ 36.00 & 48.50 & \text { " } \\ 36.00 & 48.50 & \\ 36.00 & 48.50 \\ 42.50 & 56.00 & \end{array}$

\section{SPIREA CLUMPS}

Strong undivided clumps, best forcing kinds, in following varieties:-

$$
\$ 25.00 \text { per } 100-\$ 240.00 \text { per } 1000
$$

GLADSTONE - standard white PEACH BLOSSOM - fine rosy-pink QUEEN AITXANDRA - large pink PRINCESS MARY - deep rosy-pink AMERICA - lilac-rose

GLORIA SUPERBA - clear rosy-pink, heavy clumps, $\$ 32.50$ per 100 RHINELAND - bright crimson, now DEUTSCHLAND - Pine new white

40.00

November shipment. 250 at 1000 rate.

\section{DRACAENA CANES}

These are easily grown, getting more popular every year, as they are money-makers for good growers. Note lower prices. TERMINALIS - rich crimson, marked pinis and white...... 15 $\phi$ per foot MASSANGEANA - green, with wide goldon mid-stripe...... 38 IINDENI - gold striped edges, green mid-rib.......... 35 LRS. EUGENE ANDRE - most popular bright red...........25 IORD WOLSELEY - brilliant bright red, narrow leaves.... 25ф FRAGRANS - plain green leaf, strong grower............ $22 \phi$ AMABIIIS - pink and creamy white, green base.......... 25\% BAPIISTI - striped pink and yellow on metallic green.... 30 BERTHA ANDRE - heavy broad bronze foliage............ 30 GEN. PERSHING - rosy-pink markings, green base........ 30 HARMONY - whitish margin edged pink, green base........ 30 IMPERIALIS - dark green, edged pink............... $30 \phi$ MARGUERITE STORY - light green and pink............. 30 PINK SUPERBA - narrow carmine rose foliage............ 25 PORTO RICO - reddish bronze, deep pink margin......... 30 RED CIOUD - dwarf compact grower, red foliage......... 30申 RED WING - wide spreading red leaves................ $30 \phi$ RUBY - varying shades of bronze and red............. $30 \phi$ SHEPHERDI - green, bronzy orange striped............. 30 TRICOLOR - white, pink and green colorings............25 YOUNGI - green changing to metallic bronze........... $30 \phi$ ANERLYENSIS - semi-dwarf, bronze and red............ 30 MRS. RUFUS SCOTT - bronze green with white and pink.... 30 JuNo - red and white with bronze................. 30 6

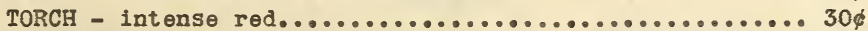

Minimum orders $10 \mathrm{ft}$. of any variety. Massangeana $2000 \mathrm{ft}$. up, and Mrs. Eugene Andre $1000 \mathrm{ft}$. up, $2 \phi$ per foot less. Will send cultural directions on request. 



\section{HYDRANGEAS}

POT-GROWN all the year, lighter plants but far better bloomers and

larger heads than the fleld-grown potted up in August.

\section{LOT \#4}

BABY BIMBENET - silvery rose

MAD. E. MOULLIERE - white

SOUV. E. CHAUTARD - pink, easy forcer GEN. DE VIBRAYE - bright rose

OTAKSA - the old reliable

E. G. HILL - clear pink, early

\section{LOT \#2}

LORELEI - carmine red

MARECHAL FOCH - deep carmine, early

PARCIFAL - red, fringed petals

HELGE - soft red

GUDRUN - clear pink

LOUIS PASTEUR - pink

\section{LOT \#3}

TROPHEE - early red

RHEINGOLD - brilliant pink

W. PFITZER - light pink

ELMAR - bright salmon, fringed petals COQUELICOT - early, deop carmine

LILIE MOULIIERE - carmine rose

\section{LOT \#1}

FREYA - glossy carmine

GERTRUDE GLAHN - dark rose

ODIN - deep carmine

MEIN IDSAL - sOPt pink

GOIIATH - rose-pink

IE CYGNZ - purest white

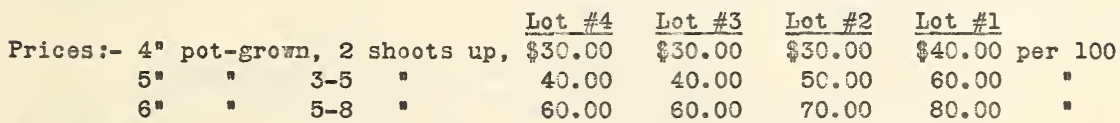

F.O.B. Maryland, including packing. Oct.-Nov. Shipment. Include in order any othor varieties you want, we can supply them.

\section{F. I. CATADIUMS}

Better bulbs in better varieties at 10 * prices. They will average $1 \frac{1}{4}-2 \frac{1}{2}$ " diameter. Winter delivery.

Good assortment of namod varieties, $\$ 14.00$ per $100 \$ 130.00$ per 1000

Mized, in same varieties

$$
13.00 \quad 120.00
$$

\section{BOAHOOD BUSHES}

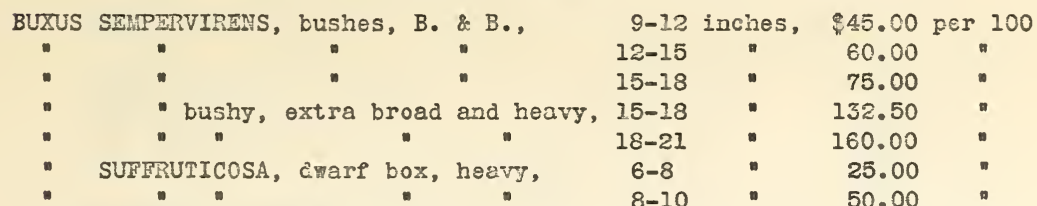

Packed and F.0.B. Maryland. Carload orcers F.O.B. Ner York. Prices on "Standards" quoted on request.

\section{FORCING FOSES}

Only the XXX grade offered - ordinary 2 year plants won't do at any price. Best varieties. Late Fall shipment. F.O.B. Ohio. ROSIS, XXX extra strong, prices except where noted, $\frac{\text { Per } 100}{\$ 36.50} \frac{\text { Per } 1000}{\$ 350.00}$

\section{Baby Rambler Type}

Red Orleans

Ellen Poulsen

Ideal

Erna Teschendorff Miss Edith Cavell

Crimson Baby

\section{Hivbrid Teas}

Radiance Columbia

Gruss an Teplitz

Lne. Ed. Herriott

Red Radiance (40\%)

Killarney Pink

Wime. Butterfly (406)
ORANEE PERFECTION (556)

Lady Readins ( $40 \%$ )

Triompho de Orleanaise

Los Angeles (406)

Hrs. Aaron Ward $(40 \phi)$

Souv. de Claudius Pernet (40\%)

\section{Hybrid Perpetuals}

Paul Neyron

Geo. Arends

Capt. Hayward

Excelsa

Dorothy Perkins

Paul's Scarlet
Frau Karl Druschki

Gen. Jacqueminot

Ulrich Brunner

\section{Climbers}

White Dorothy

Tausendschon (40\%)

Eugene Jacquet (40\%)
Hime. Albert Barbier (50\%)

MACNA CHARTA (32\%) 



\section{ENGLISH IVIES}

Field-grown plants, 24-30 inches, 3-4 branches, Per 100 $\$ 15.00 \quad \frac{\text { er } 1000}{140.00}$ Ready now.

Fiold-grown plants, established in 4 inch pots, 24-30 inches high, 3-4 branches, staked Ready in October.

$\$ 30.00 \$ 275.00$

BEGONIA and GLOXINIA BULBS

\begin{tabular}{|c|c|c|c|c|c|c|c|}
\hline T.R.BEGONIAS, & Single, & $\begin{array}{l}1-1 \frac{1 / 2}{} d \mathrm{~d} \\
11 / 2-2\end{array}$ & liam. & $\begin{array}{r}\$ 7.50 \\
11.00\end{array}$ & per 100 & $\begin{array}{r}70.00 \\
104.00\end{array}$ & per 1000 \\
\hline . & ". & $2^{\prime \prime} \&$ up & . & 13.50 & - & 128.50 & - \\
\hline & Double, & $1-1 \frac{112 n}{}$ & " & 8.25 & . & 75.00 & n \\
\hline$"$ & 1 & $1 \frac{11 / 3}{2 \prime \prime}$ & ఐ & 12.00 & " & 110.00 & " \\
\hline " & n & $2^{\prime \prime} \&$ up & , & 14.50 & n & 135.00 & $n$ \\
\hline
\end{tabular}

Colors:-Crimson, Scarlet, Rose or Pink, White, Yellow or Orange, Salmon, Mixed.

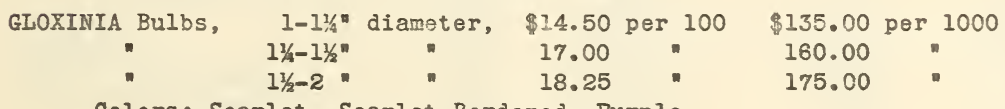

Colors:-Scarlet, Scarlet Bordered, Purple, Purple Bcrdered, White, Mized.

January delivery. Fine for pot plants for holiday trade.

\section{FORCIIHG AZAIEAS}

AZATEA INDICA, grafted,

$\begin{array}{rr}8-10 \text { inch crowns, } \$ 1.15 \text { each } \\ 10-12 \\ 12-14 \quad 2.65 \\ 14-15 \text { " } & 2.20 \%\end{array}$

Varieties:- Empress of India, Jean Haerens, Van der Cruyssen, Mrs. Fred'k Sanders, Prof. Wolters, Vervaeneana, Simon Mardxer, Vervaeneana Alba, Temperance, Niobe, Blushing Brice, Oswald de Kerchove, Jean Peeters, John Ilemelyn, Mem. Louis Van Houtte. Note:- It is best to leave the selection of varieties to us, saying what percentage of early and mid-season varieties you prefer.

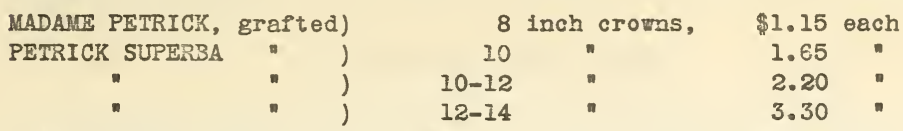

(Petrick varieties sold only with a fair proportion of cthers).

\section{Dwarf Azaleas - own roots}

\begin{tabular}{|c|c|c|c|c|c|c|c|c|}
\hline CHAS. & ENCKS & $8^{\prime \prime}$ & $\$ .90$ & each & HINODEGIP & $8=10^{n}$ & $\$ 1.15$ & each \\
\hline & - & $10^{\prime \prime}$ & 1.15 & w & n & $10-12^{\prime \prime}$ & 1.35 & 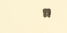 \\
\hline & $"$ & $12^{\prime \prime}$ & 1.65 & " & - & $12-14^{n}$ & 1.65 & 列 \\
\hline HEXE & (Firefly) & $8^{\prime \prime}$ & .90 & . & IORRAINI & 8 & .90 & n \\
\hline " & & 10" & 1.15 & ఐ & " & $10 \%$ & 1.15 & " \\
\hline ต & & $12^{\prime \prime}$ & 1.65 & " & " & $12^{n}$ & 1.65 & " \\
\hline
\end{tabular}

AZALEA KURUME, own roots,

$\begin{array}{rr}8 \text { inch crowns, } \$ 1.15 \text { each } \\ 10 & 1.65 \\ 12 & 2.20\end{array}$

Varieties:- Pink Pearl, Coral Bells, Christmas Cheer, and other good sorts.

\section{MANETTI STOCKS (Holland grown)}

Selected for greenhouse grafting, $1 \mathrm{rr} .5-9 \mathrm{~m} / \mathrm{m}$. December delivery. Firm price quoted later - subject to buyer's acceptance before delivery.

\section{PAPERWHITES}

The same true GRANDIFLORA type as we shipped last season - machine graded.

\begin{tabular}{|c|c|c|c|c|c|c|c|}
\hline & & & & & 1 case up & 5 cases & up \\
\hline GRANDIFLORA, & $12-13$ & $c / m$, & 1250 & per case, & $\$ 37.50$ & $\$ 37.00$ & per 1000 \\
\hline 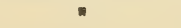 & $13-14$ & 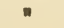 & 1000 & " & 40.00 & 39.50 & " \\
\hline " & $14-15$ & " & 900 & " & 45.00 & 44.00 & " \\
\hline n & $15-16$ & " & 800 & " & 49.00 & 48.00 & " \\
\hline
\end{tabular}

(less than case lots add 10\%)
GRAND. SOLEIL D'OR, $15 \mathrm{c} / \mathrm{m}$ up, 800 pэr case, \$9.00 per $100 \$ 82.00$ per 1000 



\section{DAFFODIL BULBS}

NORTH-WEST GROWN - same as those we shipped last season.

GOLDEN SPUR
FMPEROR
SIR WATKIN
KING ALFRED
GLORY OF SASSENHEIM
BICOLOR VICTORIA
DOUBLE VON SION
SPRING GLORY
TRESSERVE
POETAZ LAURENS KOSTBR
$\quad$ ELVIRA
ERPRSS
LUCIFER
BARRI CONSPICUOUS
POETICUS RECURVUS
$\quad$ " ORNATUS
MINISTER TALMA
WHITEWIUT
ALBATROSS
EVANGELINE
MRS. ILANGTRY
QUEEN OF THE NORTH

\begin{tabular}{rr}
\multicolumn{2}{c}{ \#1 Round } \\
per 100 & per 1000 \\
\hline 7.00 & $\$ 65.00$ \\
7.00 & 65.00 \\
6.50 & 57.00 \\
11.00 & 100.00 \\
7.50 & 70.00 \\
7.00 & 65.00 \\
6.50 & 57.50 \\
8.25 & 76.50 \\
11.00 & 100.00 \\
5.00 & 45.00 \\
5.50 & 50.00 \\
7.00 & 64.50 \\
7.00 & 65.00 \\
5.50 & 50.00 \\
4.50 & 38.00 \\
4.50 & 38.00 \\
7.50 & 70.00 \\
9.00 & 82.50 \\
7.00 & 65.00 \\
5.50 & 50.00 \\
5.00 & 43.50 \\
7.50 & 70.00
\end{tabular}

\begin{tabular}{|c|c|}
\hline per 100 & per 1000 \\
\hline$\$ 9.50$ & $\$ 90.00$ \\
\hline 9.50 & 90.00 \\
\hline 9.00 & 87.00 \\
\hline 14.50 & 137.50 \\
\hline 10.75 & 100.00 \\
\hline 9.50 & 90.00 \\
\hline 8.00 & 75.00 \\
\hline 11.50 & 105.00 \\
\hline 14.50 & 137.50 \\
\hline 7.50 & 67.50 \\
\hline 8.00 & 73.00 \\
\hline 10.00 & 92.50 \\
\hline 10.00 & 92.50 \\
\hline 8.00 & 73.00 \\
\hline 6.00 & 52.00 \\
\hline 6.50 & 58.50 \\
\hline 10.75 & 100.00 \\
\hline 12.00 & 112.00 \\
\hline 9.50 & 90.00 \\
\hline 8.00 & 75.00 \\
\hline 7.00 & 65.00 \\
\hline 10.00 & 92.50 \\
\hline
\end{tabular}

\section{DUTCH BULBS}

Only those who order DUTCH BULBS early can depend upon getting them this season. Our prediction - based on authoritative information - is that they will be even more scarce than is now generally expected. Do not throw away your opportunity to get aboar at present prices - they will likely be higher at shipping time. Write for our speclal offer on highest grade bulbs. Our prices include all charges to F.0.B. cars New York and are based on bag lots.

\section{ADDITIONAL STOCK OFFERED}

VALLEY PIPS - highest German forcing grades, new crop. GLADIOLI BULBS - all of the best varieties, northern grown only. EVERGREENS - two year old stost. BURLAP SQUARES - for balling Evergreens, bale lots.

All prices are understood packed and F.O.B. cars New York unless stated otherwise. Orders for 250 up of single 1 tems booked at 1000 prices. Terms 60 days net, less $2 \%$ cash within 10 days of involce date. Prices on all imported stock are "subject to tarlef changes".

MaEUTCHISON \& $\mathrm{CO}$. 
